\title{
Dossier: Políticas Públicas de Salud Bucal en Iberoamérica*
}

\author{
Dossier: Oral Health Public Policies in Iberian-America
}

Dossier: Políticas Públicas de Saúde Bucal na Ibero-América

\begin{abstract}
MARIANA Gabriel
Universidade de São Paulo, São Paulo, Brasil. marianagabrielodonto@gmail.com; https://orcid.org/0000-0001-8824-5827
\end{abstract}

\begin{abstract}
Maristela Honório Cayetano
Universidade Ibirapuera, São Paulo, Brasil. maricayetano@yahoo.com.br; https://orcid.org/0000$\underline{0002-0694-4171}$
\end{abstract}

Melania GonZález y Rivas

Red Odontológica Comunitaria Internacional (ROCA), Buenos Aires, Argentina. roca.org@gmail.com; https://orcid.org/0000-0003-4284-7501

\section{Marco Cornejo Ovalle \\ Universidad de Chile, Santiago, Chile. mcornejo@odontologia.uchile.cl; https://orcid.org/0000- \\ $\underline{0001-9147-5754}$}

Gabriel Jaime Otálvaro Castro 
Universidad de Antioquia, Medellín, Colombia. gjotalvaro@gmail.com; https://orcid.org/0000$\underline{0001-5110-7738}$

\section{JoRge ENRIQUE DELgAdo Troncoso}

Universidad de Pittsburgh, Pittsburgh, Pennsylvania, Estados Unidos. jed41@pitt.edu; https://orcid.org/0000-0002-1127-9516

\section{Sandra Cecilia Delgado Troncoso}

Medellín, Colombia. sandelga15@gmail.com; https://orcid.org/0000-0001-8515-5199

*Editorial

Correspondencia: $\quad$ marianagabrielodonto@gmail.com; $\quad$ maricayetano@yahoo.com.br; roca.org@gmail.com; mcornejo@ odontologia.uchile.cl; gjotalvaro@gmail.com; jed41@ pitt.edu; sandelga15@gmail.com

doi: https://doi.org/10.11144/Javeriana.uo38-80.dpps

Cómo citar: Gabriel M, Cayetano MH, González y Rivas M, Cornejo Ovalle M, Otálvaro Castro GJ, Delgado Troncoso JE, Delgado Troncoso SC. Dossier: Políticas Públicas de Salud Bucal en Iberoamérica. Univ Odontol. 2019 ene-jun; 38(80). https://doi.org/10.11144/Javeriana.uo38$\underline{80 . d p p s}$ 


\section{Palabras clave}

dossiers temáticos; Iberoamérica; observatorio; políticas públicas en salud bucal

\section{Áreas temáticas}

odontología; política pública; salud oral

\section{Keywords}

Iberian-America; observatory; oral health public policies; thematic dossiers

\section{Thematic fields}

dentistry; oral health; public policy

\section{Palavras-chave}

dossiers temáticos; Ibero-América; observatório; políticas públicas de saúde bucal

\section{Campos temáticos}

odontologia; políticas pública; saúde bucal

Tenemos el gusto de presentar a nuestros lectores el primer número de 2019 que incluye el dossier temático Políticas Públicas de Salud Bucal en Iberoamérica. Este dossier es resultado de la segunda colaboración de Universitas Odontologica con otras organizaciones. En el número 71 de 2014 publicamos los resúmenes del $5^{\circ}$ Congreso Iberoamericano de Periodoncia - 54 ${ }^{\circ}$ Seminario Anual de la Asociación Colombiana de Periodoncia y Oseointegración. 
En esta oportunidad, la publicación de este dossier tiene un sabor muy especial porque representa una construcción conjunta con el grupo de trabajo del Observatorio Iberoamericano de Políticas Públicas en Salud Bucal. Aunque recién formado, el Observatorio ha venido destacándose con una producción sólida y colaborativa. En la búsqueda de la integralidad del cuidado y de políticas públicas que apuntan a la equidad de acceso y calidad en los servicios públicos de salud, los diferentes actores involucrados en este proyecto destacan la relevancia de la salud bucal en las discusiones de los sistemas de salud de Iberoamérica a través de relatos de experiencias y políticas de salud bucal, modelos de atención y cuidado en salud, estudios epidemiológicos, así como la relación de la academia con esos servicios y políticas.

Los 10 estudios seleccionados y aquí presentados expresan el movimiento de Iberoamérica en la construcción de políticas públicas de salud bucal a partir de diferentes experiencias en los diversos países. Confirman la importancia de la evidencia científica y de compartir tales experiencias para colaborar con la toma de decisión de los gestores y los colaboradores del sistema público de salud.

Considerando la relevancia del tema, es deseable abrir más espacios para esa discusión, en el sentido de colaborar con ese intercambio de informaciones, pues aunque los países vengan desarrollando diferentes acciones de odontología en la salud pública, dicha información todavía es escasa, tanto en lo que se refiere a artículos como en la literatura gris. Otro punto importante por destacar es la necesidad de discutir las acciones en salud bucal de forma articulada a las políticas públicas y no sólo de manera puntual y aislada. 
En este contexto se espera que este dossier contribuya de manera positiva a esa construcción y sobre todo a impactar la formulación, implementación y evaluación de políticas que garanticen una oferta de la salud bucal para la población latinoamericana. 